Article

\title{
Chemotaxonomic Profiling of Canadian Alternaria Populations Using High-Resolution Mass Spectrometry
}

\author{
Megan J. Kelman ${ }^{1,2}$, Justin B. Renaud ${ }^{1}$, Keith A. Seifert ${ }^{3}$, Jonathan Mack ${ }^{3}$, \\ Ken K.-C. Yeung 2,4 (D) and Mark W. Sumarah 1,2,*(D) \\ 1 London Research and Development Center, Agriculture and Agri-Food Canada, \\ London, ON N5V 4T3, Canada; megan.kelman@canada.ca (M.J.K.); justin.renaud@canada.ca (J.B.R.) \\ 2 Department of Chemistry, University of Western Ontario, London, ON N6A 5B7, Canada; kyeung@uwo.ca \\ 3 Ottawa Research and Development Centre, Agriculture and Agri-Food Canada, \\ Ottawa, ON K1A 0C6, Canada; Keith.seifert@carleton.ca (K.A.S.); jonathan.mack2@canada.ca (J.M.) \\ 4 Department of Biochemistry, University of Western Ontario, London, ON N6A 5C1, Canada \\ * Correspondence: mark.sumarah@canada.ca; Tel.: +1-519-953-6723
}

Received: 19 May 2020; Accepted: 5 June 2020; Published: 9 June 2020

\begin{abstract}
Alternaria spp. occur as plant pathogens worldwide under field and storage conditions. They lead to food spoilage and also produce several classes of secondary metabolites that contaminate the food production chain. From a food safety perspective, the major challenge of assessing the risk of Alternaria contamination is the lack of a clear consensus on their species-level taxonomy. Furthermore, there are currently no reliable DNA sequencing methods to allow for differentiation of the toxigenic potential of these fungi. Our objective was to determine which species of Alternaria exist in Canada, and to describe the compounds they make. To address these issues, we performed metabolomic profiling using liquid chromatography high-resolution mass spectrometry (LC-HRMS) on 128 Canadian strains of Alternaria to determine their chemotaxonomy. The Alternaria strains were analyzed using principal component analysis (PCA) and unbiased $k$-means clustering to identify metabolites with significant differences $(p<0.001)$ between groups. Four populations or 'chemotypes' were identified within the strains studied, and several known secondary metabolites of Alternaria were identified as distinguishing metabolites, including tenuazonic acid, phomapyrones, and altenuene. Though species-level identifications could not be concluded for all groups through metabolomics alone, A. infectoria was able to be identified as a distinct population.
\end{abstract}

Keywords: Alternaria; high-resolution mass spectrometry; metabolomics; chemotaxonomy

\section{Introduction}

Alternaria is a cosmopolitan phytopathogenic fungal genus responsible for the spoilage of numerous agriculturally relevant crops of economic importance to Canada, including wheat, canola and tomatoes [1-4]. Non-host-specific secondary metabolites from Alternaria such as alternariol (AOH), alternariol monomethyl ether (AME), tenuazonic acid (TeA) and tentoxin (TTX) are commonly detected in processed wheat- and fruit-based commodities, including infant foods [5]. The European Union has evaluated these toxins for regulation because of their moderate cytotoxicity in vitro [5-15]. Owing to their structural similarities to the sphinganine analogue mycotoxin fumonisin, there is similar concern over the presence of AAL-toxins in tomatoes and processed tomato-based commodities [16-18].

After recent redefinitions based on whole-genome sequencing data, the genus Alternaria was divided into 27 taxonomic sections, some of which (such as Embellisia, Nimbya and Ulocladium) had previously been considered as distinct genera [19-22]. Species concepts, especially those for the most 
frequently cited species, A. alternata and A. tenuissima, were also critically re-evaluated; many species, formae speciales or pathotypes previously considered distinct because of pathogenicity to specific hosts, or morphological differences such as the length of the apical cell of the conidium, were all combined into A. alternata based on Geneological Concordance Phylogenetic Species Recognition (GCPSR) criteria [19]. It is important to note that even when the species of an Alternaria strain can be identified, the prevalence of 'conditionally-dispensable chromosomes' (CDC) that control the production of host-specific toxins can result in distinct 'pathotypes' within the same species [23]. These CDCs can disappear, or be picked up by strains through horizontal gene transfer [23-27]. Uncertainty regarding the genetic classification of Alternaria coupled with the presence of CDCs has made phylogenetic analysis and morphological characterization insufficiently reliable to predict their risk to crops or toxigenic potential $[2,28,29]$.

As defined by Frisvad, chemotaxonomy refers to "the classification and identification of filamentous fungi based on profiles of secondary metabolites" [30]. Chemotaxonomy is an especially powerful tool to understand the fungal ecology and toxigenic potential of species with CDCs, or whose properties are not fully predicted by genetic markers. Advances in metabolomics, driven by innovations in high-resolution mass spectrometry (HRMS), metabolite databases and data analysis have been beneficial for the secondary metabolite screening of filamentous fungi. Fungal culture extracts can be readily screened for hundreds of known fungal metabolites by liquid chromatography (LC)-HRMS. In a polyphasic approach with morphological and molecular information, chemical data have previously been used to differentiate among Alternaria for both large-spored, and the less morphologically distinct small-spored species, and were critical for the delineation of the species A. infectoria, which is common in wheat, and is also an opportunistic pathogen of immunocompromised humans $[2,28,29,31-36]$. Untargeted LC-HRMS analysis is essential for the characterization of chemotaxonomic groups and profiling, as targeted methods may overlook unknown but related metabolites or modified secondary metabolites that are potentially important chemotaxonomically.

The need exists for a better understanding of which species of Alternaria occur in Canada and, equally important, is the need to determine the secondary metabolites they produce. The last major survey of Canadian Alternaria was published nearly 75 years ago [37]. Due to the presence of CDCs and the uncertainty surrounding Alternaria classification, there is an enormous difficulty in estimating the toxigenic potential of strains and the risk to Canadian consumers. We have taken a chemotaxonomic approach to investigate 128 Canadian strains isolated from wheat, apples, blueberries, tomatoes and various perennial shrubs.

\section{Results}

\subsection{Principal Component Analysis (PCA) of Secondary Metabolites from Canadian Species of Alternaria}

Secondary metabolite data from the 128 strains of Alternaria analyzed by LC-HRMS in both negative and positive ionization mode were processed by PCA. Generated peak lists have been included in Supplementary A and B, and uploaded online to Metabolomics Workbench (see Supplementary). To avoid biased group assignment of the PCA plots, samples were statistically assigned into groups based on a $k$-means clustering algorithm (Figure 1A). The metabolomic analysis determined that there were four groups in negative ionization mode based on the clustering algorithm analysis and the sum of squares. Dimensions 1 and 2 represented $32.64 \%$ and $9.64 \%$ of the data variability, respectively. Clustering data were easily visualized in negative ionization mode, but it was more difficult to ascertain differences between groups 1, 2 and 4 in positive mode due to the lack of separation along the first $(24.00 \%$ of the variability) and second $(8.37 \%)$ dimensions. Although group 1 remained distinct between both ionization modes in the $k$-means clustering analysis, isolates belonging to groups 2,3 and 4 , as defined by the negative mode $k$-means analysis, became intermixed and indistinguishable in positive mode $k$-means analysis (Figure 1A). Thus, the groups generated by the $k$-means clustering algorithm in negative mode were assigned to the positive mode data to avoid unbiased visual group assignment. 

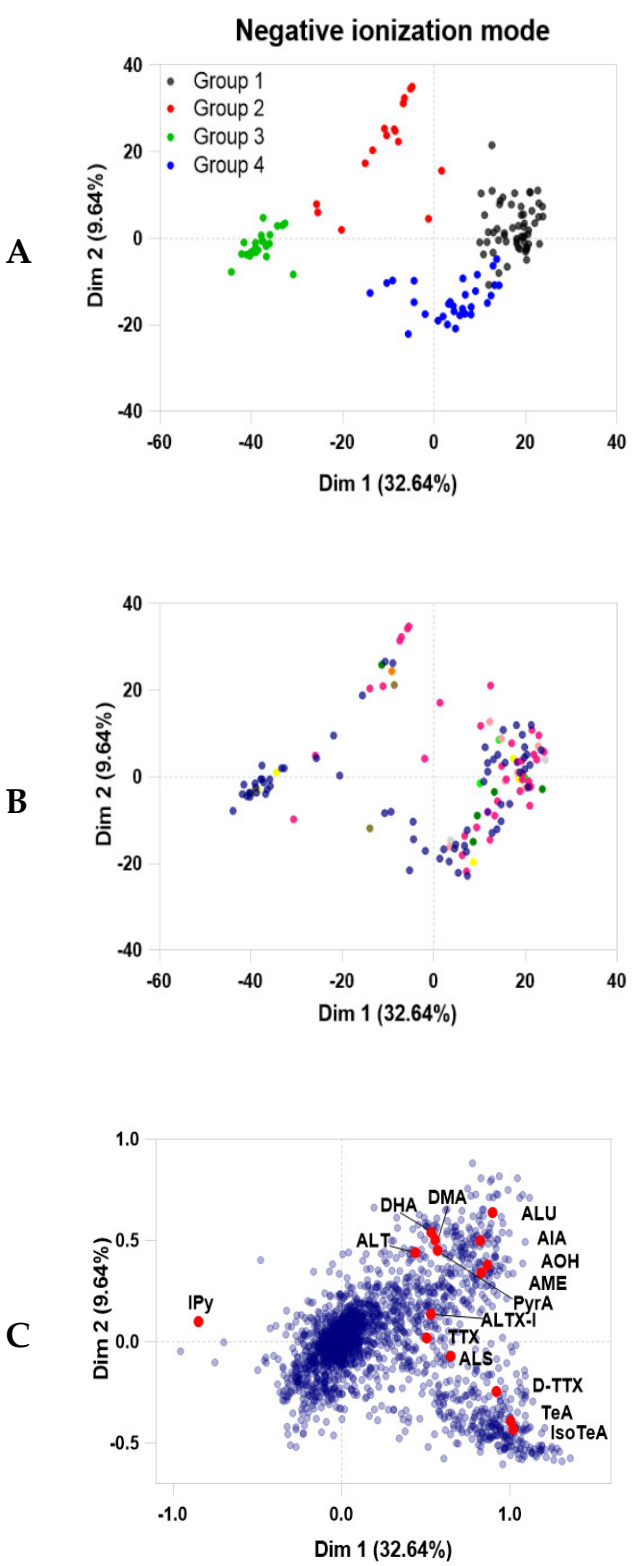
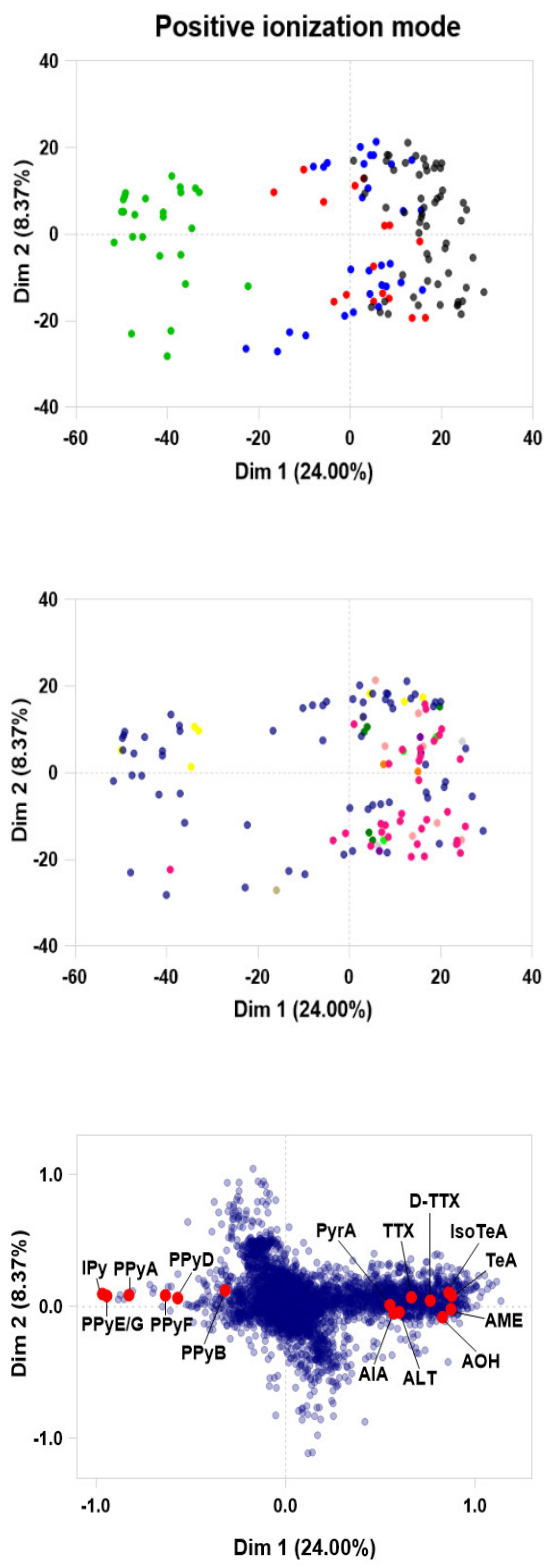

Figure 1. Principal component analysis (PCA) in both negative and positive ionization modes, where each point represents a single strain. (A) Strains in the PCA are coloured by $k$-means clustering group assignments from negative ionization mode. (B) PCA coloured by the substrate where strain was isolated. (C) Loadings plots indicating the positions of the main metabolites detected that influence the PCA separations.

Because of the potential of Alternaria species to produce host-specific toxins, we investigated whether the populations observed were related to the substrate from which they were isolated. Individual isolates are coloured by the substrate they were collected from in the PCA plots (Figure 1B) and a host distribution is shown in Figure 2. Group 3 primarily consisted of isolates from grain (80\%) however, more host diversity was observed in Groups 1, 2 and 4 (Figure 2). The specific metabolites from each of the assigned populations were further investigated, and are reported in Table 1. All isolates were screened for the production of AAL-toxins, but none were detected. 


\section{Group 1 Group 2 Group 3 Group 4}
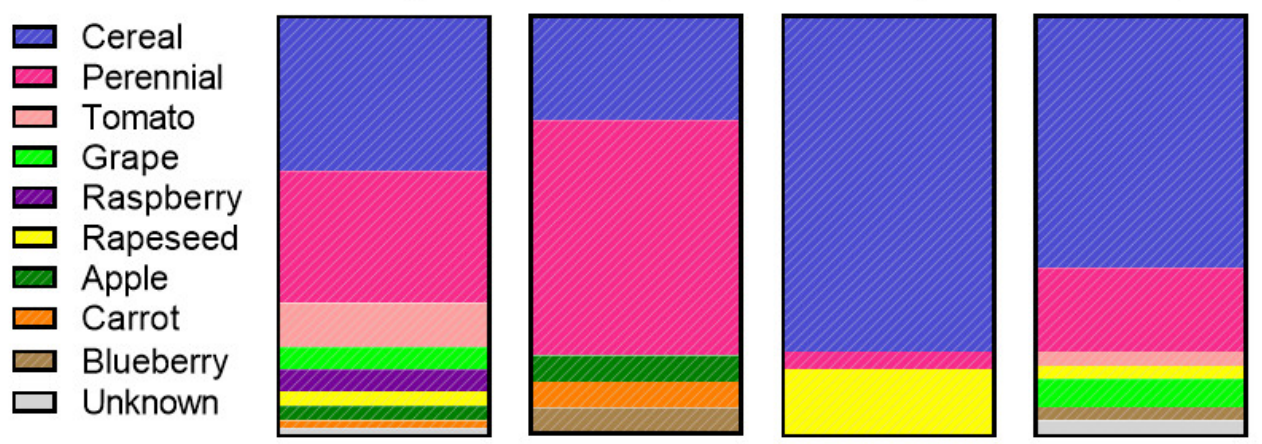

Figure 2. Substrate distribution within groups assigned by $k$-means clustering.

Table 1. Known Alternaria metabolites detected in 128 Canadian strains of Alternaria separated by their population, and percentage of isolates producing them.

\begin{tabular}{|c|c|c|c|c|c|c|}
\hline \multirow[b]{2}{*}{ Metabolite } & \multirow[b]{2}{*}{ Abbr. } & \multirow[b]{2}{*}{ RT (min) } & \multicolumn{4}{|c|}{ Populations of Canadian Strains (\% of Strains) } \\
\hline & & & $\begin{array}{l}\text { Group } 1 \\
(n=57)\end{array}$ & $\begin{array}{c}\text { Group } 2 \\
(n=16)\end{array}$ & $\begin{array}{l}\text { Group } 3 \\
(n=25)\end{array}$ & $\begin{array}{c}\text { Group } 4 \\
(n=30)\end{array}$ \\
\hline infectopyrone & IPy & 3.03 & - & - & 100 & - \\
\hline phomapyrone A & PPyA & 3.73 & - & - & 100 & - \\
\hline phomapyrone B & PPyB & 2.83 & - & - & 85 & - \\
\hline phomapyrone D & PPyD & 3.04 & - & - & 100 & - \\
\hline phomapyrone $\mathrm{E} / \mathrm{G}$ & PPyE/G & 2.99 & - & - & 100 & - \\
\hline phomapyrone $\mathrm{F}$ & PPyF & 3.01 & - & - & 50 & - \\
\hline tenuazonic acid & $\mathrm{TeA}$ & 3.05 & 100 & - & - & 100 \\
\hline iso-tenuazonic acid derivative & IsoTeA & 2.81 & 100 & - & - & 100 \\
\hline alternariol * & $\mathrm{AOH}$ & 3.1 & 100 & 94 & - & 91 \\
\hline alternariol monomethyl ether * & AME & 3.53 & 100 & 100 & - & 94 \\
\hline altenusin * & ALU & 2.97 & 100 & 71 & - & 55 \\
\hline desmethylaltenusin * & DMA & 2.75 & 23 & 18 & - & - \\
\hline dehydroaltenusin * & DHA & 3.26 & 72 & 41 & - & 9 \\
\hline alternarienoic acid * & $\mathrm{AlA}$ & 2.71 & 100 & 71 & - & 30 \\
\hline altenuene & ALT & 2.87 & 70 & 53 & - & - \\
\hline altechromone A & ALCA & 2.82 & 79 & 53 & - & 30 \\
\hline altechromone B & ALCB & 2.72 & 98 & 82 & - & 12 \\
\hline altertoxin I* & ALTX-I & 2.53 & 33 & 59 & - & 36 \\
\hline altertoxin II * & ALTX-II & 2.93 & 16 & 35 & - & 30 \\
\hline altertoxin III * & ALTX-III & 3.27 & 26 & 18 & - & 9 \\
\hline tentoxin & TTX & 3.11 & 30 & 29 & - & 21 \\
\hline dihydrotentoxin & D-TTX & 3.13 & 33 & 24 & - & 15 \\
\hline altersetin * & ALS & 4.37 & 68 & 53 & - & 67 \\
\hline pyrenochaetic acid A & PyrA & 2.63 & 93 & 71 & - & 21 \\
\hline
\end{tabular}

* Analysis from negative ionization mode data.

\subsection{Statistical and Metabolomic Analysis of Detected Canadian Alternaria Populations}

The loading plots (Figure 1C) show the individual metabolites responsible for the strain coordinates within the PCA plots, and have values ranging from -1 to +1 along both dimensions. Metabolite features that are shared by multiple chemotype groups account for less variance and weight in the PCA, have negligible (near 0) loadings, and appear close to the origin. Conversely, metabolites with the strongest influence or effects on the components in the dataset have larger values, close to either +1 or -1 . Larger loading values show the metabolite directionality in the PCA plot, corresponding to the potential presence or absence of that metabolite within a particular group. Metabolites with the strongest influence were further investigated for their statistical differences between each group using 
the Kruskal-Wallis test. Calculated $p$ values were corrected based on post-hoc Benjamini-Hochberg (BH) false discovery rate (FDR) correction.

The metabolites responsible for the majority of the variance in the PCA included the phomapyrones $\left(p<0.001\right.$, FDR corrected, see Supplementary A and B) and infectopyrone (IPy, $\left.p=2.03 \times 10^{-12}\right)$, the amino acid derived tenuazonic acid (TeA, $p=4.80 \times 10^{-17}$ ) and its valine-substituted iso-tenuazonic acid, (IsoTeA, $p=1.61 \times 10^{-16}$ ), and several secondary metabolites known to be produced by Alternaria. Metabolites that are directly opposite to each other along the diagonal of the loadings plot are negatively correlated. For instance, the infectopyrones and phomapyrones were observed to be strongly correlated to each other, but were negatively correlated to the other dibenzopyrones, such as alternariol $(\mathrm{AOH}$, $\left.p=1.95 \times 10^{-16}\right)$, and alternariol monomethyl ether (AME, $p=5.69 \times 10^{-17}$ ).

Table 1 shows the known secondary metabolites produced by Alternaria, and which chemotaxonomic groups they were detected in. Though the list is not exhaustive, it represents the breadth of known and well characterized secondary metabolites, either through analytical standards, or from published LC-MS/MS transitions. Both known and unknown significant $(p<0.001)$ metabolites have been included in Supplementary A and B, and through Metabolomics Workbench, (included in Supplementary). Most of the dibenzopyrones and commonly detected secondary metabolites of Alternaria are capable of ionizing in both positive and negative ionization modes, but the majority of phomapyrones, which are commonly associated with A. infectoria, ionized best in the positive ionization mode (see Supplementary). Ionization in positive mode was only able to distinguish group 3 from the other groups, as it did not share metabolites (Table 1). The PCA separation across both dimensions was best observed in the negative ionization mode. In addition to the better ionization of dibenzopyrones, the variability across the first dimension was predominantly due to the presence or absence of tenuazonic acid (TeA) (Figure 1C). Groups 2 and 3, which cluster in negative dimension 1, do not produce TeA. Both of these groups are also differentiated from each other across the second dimension due to the differentiating metabolites produced solely by the isolates in group 3 , such as infectopyrone (IPy). Though group 2 does not produce TeA, it does share other common metabolites with groups 1 and 4, including alternariol $(\mathrm{AOH})$ and alternariol monomethyl ether (AME) (Table 1). Variation in the second dimension of the PCA plots (Figure 1A), especially between groups 1 and 4, was largely influenced by abundances in the detected peak areas. Both groups 1 and 4 include many of the same metabolites, but group 1 tended to have higher abundances of those shared metabolites, (Supplementary A and B). The major difference between groups 1 and 4 was that group 4 did not produce altenuene or desmethylaltenusin.

Of the 4170 metabolite features detected in the positive ionization mode data, there were 1593 significant metabolites $(p<0.001)$ after applying the Kruskal-Wallis test using the assigned groups from the $k$-means clustering (Supplementary A). Similarly, there were 2198 metabolite features detected in negative ionization mode, and 1060 were significant $(p<0.001)$ (Supplementary B).

\section{Discussion}

There was little correlation between the chemotype group and the substrate of origin; a wide substrate diversity was observed within PCA groups 1,2 and 4. Population 3 had the most unique metabolite profile. It did not produce the dibenzopyrones $\mathrm{AOH}$ and $\mathrm{AME}$, nor tenuazonic acid. It was however, the only population that produced infectopyrone and phomapyrones. This population was isolated predominately from cereal (Figure 2) as well as some perennial and rapeseed. Based on the substrate of isolation and the production of infectopyrones, this population is likely comprised of the $A$. infectoria species group [31]. It is unsurprising that $A$. infectoria was distinguished through metabolomics methods, as it was previously reported to be a very distinct section (section Infectoriae) within Alternaria [36,38].

The remaining population groups, 1, 2 and 4, all produced varying amounts of dibenzopyrones; however, the major differentiating feature was the presence or absence of TeA, (Supplementary A and B). TeA was produced by groups 1 and 4 , but absent in group 2 . TeA production has been previously 
linked to a non-ribosomal peptide synthetase and polyketide synthase (PKS) hybrid enzyme, of which the ketosynthase tenuazonic acid synthetase (TAS1 KS) domain was reported to be imperative for the production of TeA [39]. Similarly, the production of alternariol (AOH) has been correlated with the expression levels from the polyketide synthase gene, pksJ [40].

As PCA is particularly sensitive to the relative abundances of metabolites, it was challenging to discern many differences between groups 1, 2 and 4. Andersen (2015) previously reported that production of metabolites was inconsistent between isolates, even for the common dibenzopyrones $\mathrm{AOH}$ and AME, making differentiation by metabolomics alone much more difficult [2]. It is unclear whether the observed separation of the 128 Alternaria isolates is a consequence of species-level differences, PKS mutations, gene expression levels, or if dispensable chromosomes coding for host-specific toxins were present. There are also extenuating environmental conditions, because there are apparently marked differences between Canadian strains, and other strains of A. infectoria isolated from around the world; Canadian strains did not produce the novae-zelandins, as reported previously for this species $[2,29,31]$. The apparent lack of substrate specificity within the groups suggests that the chemotypes identified herein could be detected on numerous commercial commodities. The substantial population of $A$. infectoria present on wheat and cereal crops indicates that its secondary metabolites may need to be monitored alongside the other food-relevant Alternaria secondary metabolites in produce and processed commodities. While the European Food Safety Authority is considering regulating several Alternaria secondary metabolites, including TeA and TTX, there are currently no regulatory limits.

\section{Materials and Methods}

\subsection{Fungal Material and Identification}

The 128 strains of Alternaria spp. studied in this work were obtained from the Canadian Collection of Fungal Cultures (CCFC) in Ottawa, Ontario, the Canadian Grain Commission (CGC) in Winnipeg, Manitoba or isolated from local sources. The majority of the strains were isolated from food-relevant crops, including grain, apples, tomatoes and grapes, although several strains were isolated from various perennial shrubs, (Table S1, Supplementary C). Individual isolated strains not obtained from CCFC or CGC were identified to the genus level based on morphology. To identify the species, partial DNA sequences for either RNA polymerase II second largest subunit (RPB2) or complete sequences of rDNA internal transcribed spacers (ITS) were determined for 108 of the strains using the primers, amplification and sequencing parameters of Woudenberg et al. [19]. The data were examined by J. Woudenberg of the Westerdijk Fungal Biodiversity Institute, Utrecht, the Netherlands in comparison with her reference data (Supplementary C).

\subsection{Agar Plug Extraction}

Potato dextrose agar (PDA) was selected for metabolomic analysis due to the large diversity of metabolites produced in comparison to the other culture media tested (data not shown). Strains were transferred as 3-point inoculations onto PDA plates (Sigma Aldrich, St. Louis, MO, USA). Cultures were incubated at $25^{\circ} \mathrm{C}$ in darkness for seven days, during which the majority of the strains reached approximately $4 \mathrm{~cm}$ in diameter. Following incubation, six agar plugs were removed from each 3-point inoculum using a $6 \mathrm{~mm}$ cork borer, followed by extraction by ethyl acetate containing $1 \%$ formic acid (Sigma Aldrich, St. Louis, MO, USA). Prior to LC-MS screening, extracts were dried under nitrogen before reconstitution in acetonitrile, and filtration with $0.45 \mu \mathrm{m}$ PTFE syringe filters (ChromeSpec) into vials $[35,41]$.

\subsection{LC-HRMS Analysis}

High-resolution mass spectrometry (HRMS) data were obtained using a Thermo Q-Exactive Quadrupole Orbitrap Mass Spectrometer coupled to an Agilent 1290 HPLC. Chromatography and mass 
spectrometry conditions for both polar and nonpolar compounds were previously optimized [16,42]. For chromatographic separation, a Zorbax Eclipse Plus RRHD C18 column $(2.1 \times 50 \mathrm{~mm}, 1.8 \mu \mathrm{m}$; Agilent) was maintained at $35^{\circ} \mathrm{C}$ using mobile phases comprised of water with $0.1 \%$ formic acid (A), and acetonitrile with $0.1 \%$ formic acid (B) (Optima grade, Fisher Scientific, Lawn, NJ, USA). Mobile phase B was held at $0 \%$ for $30 \mathrm{~s}$, and increased to $100 \%$ over three and a half minutes. B was held at $100 \%$ for 1 and a half minutes, before returning to $\%$ B in $30 \mathrm{~s}$. Injections were made at a volume of $2 \mu \mathrm{L}$ and a flow rate of $0.3 \mathrm{~mL} / \mathrm{min}$ was used. The following conditions were used for negative HESI for full MS: capillary voltage, $3.7 \mathrm{kV}$; capillary temperature, $400{ }^{\circ} \mathrm{C}$; sheath gas, 17.00 units; auxiliary gas, 8.00 units; probe heater temperature, $450{ }^{\circ} \mathrm{C}$; S-Lens RF level, 45.00; AGC target, 1e6; maximum injection time (IT), $512 \mathrm{~ms}$; scan range $\mathrm{m} / \mathrm{z}$ 100-1200. Similar conditions were used for positive HESI, but with a capillary voltage of $3.9 \mathrm{kV}$. HRMS data for both positive and negative full MS data were acquired at a resolution of 140,000 and maximum IT of 500 ms. Lock masses of Di-n-butyl phthalate (DBP) and sodiated formic acid (FA) dimer were monitored in positive and negative ionization modes respectively to account for instrumental drift.

\subsection{Principal Component Analysis (PCA) and k-Means Clustering Analysis}

Raw HRMS data generated in both positive and negative mode were converted to mzml files and centroided prior to XCMS processing in R (r-project.org) to generate peak lists (see Supplementary) [43-45]. The applied XCMS conditions for the production of the peak lists are listed in Supplementary C. Sample carry-over was corrected according to the maximum peak areas detected in the blanks. Due to the minfrac XCMS settings, lower intensity metabolites not present in at least $25 \%$ of the sample dataset were not counted as peaks. Thus, the presence or absence of metabolites was confirmed through analysis of the raw data files. Any metabolites from the peak list with peak areas equal to zero were replaced with $2 / 3$ of the minimum peak area value of all metabolites detected [46]. Following zero replacements, a log transformation of the peak areas was performed. Pareto scaling was used to adjust for fold differences between metabolites during PCA generation using MetabolAnalyze and FactoMineR packages. Unsupervised groups from the PCA were assigned by $k$-means clustering analysis using scripts adapted for R [47]. From the logged peak lists of the metabolite datasets, the appropriate $k$-means cluster was investigated for up to six groups using the elbow method of the within sum of squares (WSS) plot. Variations identified by PCA and $k$-means clustering analysis between samples were investigated through statistical analysis of metabolomic data.

\subsection{Metabolomic Analysis}

Each metabolite in the peak list was investigated for its significance using the Kruskal-Wallis test with post-hoc Benjamini-Hochberg (BH) False Discovery Rate (FDR) correction with a threshold value of $p<0.01$ to account for multiple hypothesis testing. Metabolites with FDR corrected threshold $p$ values $<0.001$ and $\log 2$ of the average peak area values $>1$ were further investigated within each group assignment. Significant metabolites were confirmed through comparison to authentic standards, or to the published literature MS/MS data. All unknown metabolites were searched using SciFinder and Antibase 2013. 44 of the 128 strains were subsequently re-cultured, re-extracted and re-analyzed, as described above, to ensure that the metabolomic analyses were consistent.

\section{Conclusions}

Four main chemotaxonomic populations of Canadian Alternaria were identified, including A. infectoria, which was largely present on cereals and rapeseed. Substrate had no apparent effect on chemotaxonomy, though there were marked metabolite differences between Canadian Alternaria strains and other reported strains from around the world. Distinguishing secondary metabolites including IPy, the phomapyrones, ALT, DMA and TeA may assist in future organizations of the genus. Identification of Alternaria species and linking them to secondary metabolite production remains a great challenge. More work is needed from a mycology perspective with respect to their taxonomy. 
Metabolomic profiling provides another key component to help aid in identification of Alternaria, and more importantly provides critical insight into the toxigenic potential of these strains, and the risk to consumers.

Supplementary Materials: The following are available online at http://www.mdpi.com/2218-1989/10/6/238/s1. Raw data and peak list files are available at the NIH Common Fund's National Metabolomics Data Repository (NMDR) website, the Metabolomics Workbench, https://www.metabolomicsworkbench.org where they have been assigned Study ID ST001370. They can be accessed directly using Project DOI 10.21228/M8Q114. Supplementary A: List of 1593 significant metabolites by their $m / z(p<0.001)$ as calculated by the Kruskal-Wallis test from the positive ionization mode dataset; Supplementary B: List of 1060 significant metabolites by their $m / z(p<0.001)$ as calculated by the Kruskal-Wallis test from the negative ionization mode dataset; Supplementary C: List of 128 Alternaria strains used in the metabolomic analysis, including RPB2 primer analysis \& xcms conditions used in R.

Author Contributions: Conceptualization, M.W.S., K.A.S.; Methodology, M.J.K., J.M.; Software, M.J.K., J.B.R.; Validation, M.J.K., M.W.S., J.B.R.; Formal analysis, M.J.K.; Investigation, M.J.K.; Resources, K.A.S., K.K.-C.Y.; Data curation, M.J.K.; Writing—original draft preparation, M.J.K.; writing—review and editing, M.J.K., M.W.S., J.B.R., K.A.S., K.K.-C.Y.; Supervision, M.W.S., K.K.-C.Y.; Project administration, M.W.S.; Funding acquisition, M.W.S, K.A.S. All authors have read and agree to the published version of the manuscript.

Funding: This research was funded by an AAFC grant to M.W.S. and K.A.S.

Acknowledgments: The authors thank A. McMillan (Cleveland Clinic) for assistance with data processing and J. Woudenberg (CBS) for sequencing help.

Conflicts of Interest: The authors declare no conflict of interest.

\section{References}

1. Patriarca, A.; Azcarate, M.P.; Terminiello, L.; Fernández Pinto, V. Mycotoxin production by Alternaria strains isolated from Argentinean wheat. Int. J. Food Microbiol. 2007, 119, 219-222. [CrossRef]

2. Andersen, B.; Nielsen, K.F.; Fernández Pinto, V.; Patriarca, A. Characterization of Alternaria strains from Argentinean blueberry, tomato, walnut and wheat. Int. J. Food Microbiol. 2015, 196, 1-10. [CrossRef] [PubMed]

3. Tralamazza, S.M.; Piacentini, K.C.; Iwase, C.H.T.; Rocha, L.d.O. Toxigenic Alternaria species: Impact in cereals worldwide. Curr. Opin. Food Sci. 2018, 23, 57-63. [CrossRef]

4. Ayer, W.A.; Pena-Rodriguez, L.M. Metabolites produced by Alternaria brassicae, the black spot pathogen of canola. Part 1, the phytotoxic components. J. Nat. Prod. 1987, 50, 400-407. [CrossRef]

5. European Food Safety Authority; Arcella, D.; Eskola, M.; Ruiz, J.A.G. Dietary exposure assessment to Alternaria toxins in the European population. EFSA J. 2016, 14. [CrossRef]

6. European Food Safety Authority. Scientific opinion on the risks for animal and public health related to the presence of Alternaria toxins in feed and food. EFSA J. 2011, 9, 97.

7. Harimoto, Y.; Tsuge, T.; Akimitsu, K.; Ohtani, K.; Otani, H.; Egusa, M.; Kodama, M.; Akagi, Y.; Yamamoto, M. Host-selective toxins produced by the plant pathogenic fungus Alternaria alternata. FEMS Microbiol. Rev. 2013, 37, 44-66.

8. Takaoka, S.; Kurata, M.; Harimoto, Y.; Hatta, R.; Yamamoto, M.; Akimitsu, K.; Tsuge, T. Complex regulation of secondary metabolism controlling pathogenicity in the phytopathogenic fungus Alternaria alternata. New Phytol. 2014, 202, 1297-1309. [CrossRef] [PubMed]

9. Schrader, T.J.; Cherry, W.; Soper, K.; Langlois, I.; Vijay, H.M. Examination of Alternaria alternata mutagenicity and effects of nitrosylation using the ames salmonella test. Teratog. Carcinog. Mutagenesis 2001, 21, 261-274. [CrossRef]

10. Brugger, E.-M.; Wagner, J.; Schumacher, D.M.; Koch, K.; Podlech, J.; Metzler, M.; Lehmann, L. Mutagenicity of the mycotoxin alternariol in cultured mammalian cells. Toxicol. Lett. 2006, 164, 221-230. [CrossRef]

11. Scott, P.M.; Zhao, W.; Feng, S.; Lau, B.P.-Y. Alternaria toxins alternariol and alternariol monomethyl ether in grain foods in Canada. Mycotoxin Res. 2012, 28, 261-266. [CrossRef] [PubMed]

12. Van de Perre, E.; Deschuyffeleer, N.; Jacxsens, L.; Vekeman, F.; Van Der Hauwaert, W.; Asam, S.; Rychlik, M.; Devlieghere, F.; De Meulenaer, B. Screening of moulds and mycotoxins in tomatoes, bell peppers, onions, soft red fruits and derived tomato products. Food Control. 2014, 37, 165-170. [CrossRef] 
13. Lau, B.P.Y.; Scott, P.M.; Lewis, D.A.; Kanhere, S.R.; Cléroux, C.; Roscoe, V.A. Liquid chromatography-mass spectrometry and liquid chromatography-tandem mass spectrometry of the Alternaria mycotoxins alternariol and alternariol monomethyl ether in fruit juices and beverages. J. Chromatogr. A 2003, 998, 119-131. [CrossRef]

14. Asam, S.; Rychlik, M. Potential health hazards due to the occurrence of the mycotoxin tenuazonic acid in infant food. Eur. Food Res. Technol. 2013, 236, 491-497. [CrossRef]

15. Rychlik, M.; Lepper, H.; Weidner, C.; Asam, S. Risk evaluation of the Alternaria mycotoxin tenuazonic acid in foods for adults and infants and subsequent risk management. Food Control. 2016, 68, 181-185. [CrossRef]

16. Renaud, J.B.; Kelman, M.J.; Qi, T.F.; Seifert, K.A.; Sumarah, M.W. Product ion filtering with rapid polarity switching for the detection of all fumonisins and AAL-toxins. Rapid Commun. Mass Spectrom. 2015, 29, 2131-2139. [CrossRef]

17. Shier, W.T.; Abbas, H.K.; Mirocha, C.J. Toxicity of the mycotoxins Fumonisins B1 and B2 and Alternaria alternata f. sp. lycopersici toxin (AAL) in cultured mammalian cells. Mycopathologia 1991, 116, 97-104. [CrossRef]

18. IARC Working Group on the Evaluation of Carcinogenic Risks to Humans. Some Traditional Herbal Medicines, Some Mycotoxins, Naphthalene and Styrene; IARC monographs on the evaluation of carcinogenic risks to humans; IARC: Lyon, France, 2002.

19. Woudenberg, J.H.C.; Groenewald, J.Z.; Binder, M.; Crous, P.W. Alternaria redefined. Stud. Mycol. 2013, 75, 171-212. [CrossRef]

20. Lawrence, D.P.; Gannibal, P.B.; Peever, T.L.; Pryor, B.M. The sections of Alternaria: Formalizing species-group concepts. Mycologia 2013, 105, 530-546. [CrossRef]

21. Woudenberg, J.H.C.; Seidl, M.F.; Groenewald, J.Z.; de Vries, M.; Stielow, J.B.; Thomma, B.P.H.J.; Crous, P.W. Alternaria section alternaria: Species, formae speciales or pathotypes? Stud. Mycol. 2015, 82, 1-21. [CrossRef]

22. Lawrence, D.P.; Rotondo, F.; Gannibal, P.B. Biodiversity and taxonomy of the pleomorphic genus Alternaria. Mycol. Prog. 2015, 15, 3. [CrossRef]

23. Hatta, R.; Ito, K.; Hosaki, Y.; Tanaka, T.; Tanaka, A.; Yamamoto, M.; Akimitsu, K.; Tsuge, T. A conditionally dispensable chromosome controls host-specific pathogenicity in the fungal plant pathogen Alternaria alternata. Genetics 2002, 161, 59-70.

24. Masunaka, A.; Ohtani, K.; Peever, T.L.; Timmer, L.W.; Tsuge, T.; Yamamoto, M.; Yamamoto, H.; Akimitsu, K. An isolate of Alternaria alternata that is pathogenic to both tangerines and rough lemon and produces two host-selective toxins, ACT- and ACR-toxins. Phytopathology 2005, 95, 241-247. [CrossRef]

25. Akagi, Y.; Taga, M.; Yamamoto, M.; Tsuge, T.; Fukumasa-Nakai, Y.; Otani, H.; Kodama, M. Chromosome constitution of hybrid strains constructed by protoplast fusion between the tomato and strawberry pathotypes of Alternaria alternata. J. Gen. Plant Pathol. 2009, 75, 101. [CrossRef]

26. Salamiah; Akamatsu, H.; Fukumasa-Nakai, Y.; Otani, H.; Kodama, M. Construction and genetic analysis of hybrid strains between apple and tomato pathotypes of Alternaria alternata by protoplast fusion. J. Gen. Plant Pathol. 2001, 67, 97-105. [CrossRef]

27. Salamiah; Fukumasa-Nakai, Y.; Akamatsu, H.; Otani, H.; Kohmoto, K.; Kodama, M. Genetic analysis of pathogenicity and host-specific toxin production of Alternaria alternata tomato pathotype by protoplast fusion. J. Gen. Plant Pathol. 2001, 67, 7-14.

28. da Cruz Cabral, L.; Rodriguero, M.; Stenglein, S.; Fog Nielsen, K.; Patriarca, A. Characterization of small-spored Alternaria from Argentinean crops through a polyphasic approach. Int. J. Food Microbiol. 2017, 257, 206-215. [CrossRef]

29. Patriarca, A.; da Cruz Cabral, L.; Pavicich, M.A.; Nielsen, K.F.; Andersen, B. Secondary metabolite profiles of small-spored Alternaria support the new phylogenetic organization of the genus. Int. J. Food Microbiol. 2019, 291, 135-143. [CrossRef]

30. Frisvad, J.C.; Andersen, B.; Thrane, U. The use of secondary metabolite profiling in chemotaxonomy of filamentous fungi. Mycol. Res. 2008, 112, 231-240. [CrossRef]

31. Andersen, B.; Sørensen, J.L.; Nielsen, K.F.; van den Ende, B.G.; de Hoog, S. A polyphasic approach to the taxonomy of the Alternaria infectoria species-group. Fungal Genet. Biol. 2009, 46, 642-656. [CrossRef]

32. Andersen, B.; Thrane, U. Differentiation of Alternaria infectoria and Alternaria alternata based on morphology, metabolite profiles, and cultural characteristics. Can. J. Microbiol. 1996, 42, 685-689. [CrossRef]

33. Andersen, B.; Krøger, E.; Roberts, R.G. Chemical and morphological segregation of Alternaria alternata, A. gaisen and A. longipes. Mycol. Res. 2001, 105, 291-299. [CrossRef] 
34. Andrew, M.; Peever, T.L.; Pryor, B. An expanded multilocus phylogeny does not resolve morphological species within the small-spored Alternaria species complex. Mycologia 2009, 101, 95-109. [CrossRef]

35. Andersen, B.; Dongo, A.; Pryor, B.M. Secondary metabolite profiling of Alternaria dauci, A. porri, A. solani, and A. Tomatophila. Mycol. Res. 2008, 112, 241-250. [CrossRef]

36. Somma, S.; Amatulli, M.T.; Masiello, M.; Moretti, A.; Logrieco, A.F. Alternaria species associated to wheat black point identified through a multilocus sequence approach. Int. J. Food Microbiol. 2019, 293, 34-43. [CrossRef] [PubMed]

37. Groves, J.W.; Skolko, A.J. Notes on seed-borne fungi: Ii. Alternaria. Can. J. Res. 1944, 22c, 217-234. [CrossRef]

38. Pryor, B.M.; Bigelow, D.M. Molecular characterization of Embellisia and Nimbya species and their relationship to Alternaria, Ulocladium and Stemphylium. Mycologia 2003, 95, 1141-1154. [CrossRef] [PubMed]

39. Yun, C.-S.; Motoyama, T.; Osada, H. Biosynthesis of the mycotoxin tenuazonic acid by a fungal NRPS-PKS hybrid enzyme. Nat. Commun. 2015, 6, 8758. [CrossRef]

40. Saha, D.; Fetzner, R.; Burkhardt, B.; Podlech, J.; Metzler, M.; Dang, H.; Lawrence, C.; Fischer, R. Identification of a polyketide synthase required for alternariol (AOH) and alternariol-9-methyl ether (AME) formation in Alternaria alternata. PLoS ONE 2012, 7, e40564. [CrossRef]

41. Frisvad, J.C.; Smedsgaard, J.; Samson, R.A.; Larsen, T.O.; Thrane, U. Fumonisin B2 production by Aspergillus niger. J. Agric. Food Chem. 2007, 55, 9727-9732. [CrossRef]

42. Kelman, M.J.; Renaud, J.B.; Seifert, K.A.; Mack, J.; Sivagnanam, K.; Yeung, K.K.-C.; Sumarah, M.W. Identification of six new Alternaria sulfoconjugated metabolites by high-resolution neutral loss filtering. Rapid Commun. Mass Spectrom. 2015, 29, 1805-1810. [CrossRef]

43. Smith, C.A.; Want, E.J.; O'Maille, G.; Abagyan, R.; Siuzdak, G. Xcms: Processing mass spectrometry data for metabolite profiling using nonlinear peak alignment, matching, and identification. Anal. Chem. 2006, 78, 779-787. [CrossRef]

44. Tautenhahn, R.; Böttcher, C.; Neumann, S. Highly sensitive feature detection for high resolution LC/MS. BMC Bioinform. 2008, 9, 504. [CrossRef]

45. Want, E.J.; Benton, H.P.; Ebbels, T.M.D. Correction of Mass Calibration Gaps in Liquid Chromatography-Mass Spectrometry Metabolomics Data. Bioinformatics 2010, 26, 2488-2489.

46. McMillan, A.; Rulisa, S.; Sumarah, M.; Macklaim, J.M.; Renaud, J.; Bisanz, J.E.; Gloor, G.B.; Reid, G. A multi-platform metabolomics approach identifies highly specific biomarkers of bacterial diversity in the vagina of pregnant and non-pregnant women. Sci. Rep. 2015, 5, 14174. [CrossRef]

47. MacQueen, J. Some Methods for Classification and Analysis of Multivariate Observations, Proceedings of the Fifth Berkeley Symposium on Mathematical Statistics and Probability; University of California Press: Berkeley, CA, USA, 1967; Volume 1, pp. 281-297. 